\title{
Power Supplies for High Energy Particle Accelerators
}

\author{
Pranab Kumar Dey
}

Received: 31 March 2013/Accepted: 2 March 2015/Published online: 23 May 2015

(C) The Institution of Engineers (India) 2015

\begin{abstract}
The on-going research and the development projects with Large Hadron Collider at CERN, Geneva, Switzerland has generated enormous enthusiasm and interest amongst all to know about the ultimate findings on 'God's Particle'. This paper has made an attempt to unfold the power supply requirements and the methodology adopted to provide the stringent demand of such high energy particle accelerators during the initial stages of the search for the ultimate particles. An attempt has also been made to highlight the present status on the requirement of power supplies in some high energy accelerators with a view that, precautionary measures can be drawn during design and development from earlier experience which will be of help for the proposed third generation synchrotron to be installed in India at a huge cost.
\end{abstract}

Keyword High energy accelerators .

Supplies for fluctuating loads .

Power supplies for accelerators

\section{Introduction}

The latest result of the experiments at Large Hadron Collider (LHC), at Geneva, Switzerland, where the physicists are engaged in the fundamental research has generated tremendous enthusiasm and interests among the scientific community. The LHC, the biggest machine in the world to carry out the research in nuclear science has opened new frontier of knowledge in various fields. Similar situation

P. K. Dey $(\square)$

Hindustan Copper Limited, Ghatsila, Jharkhand, India

e-mail: pkdasso@yahoo.co.in experienced during the early sixties at the Rutherford High energy Laboratory at Chilton, Berkshire [1], England, over the development of $7 \mathrm{GeV}$ proton synchrotron, has been discussed in this paper. The size and technology involved in building the proton synchrotron National Institute Machine Radiating on Downs (Nimrod) in 1957 of Rutherford High Energy Laboratory sounds miniscule today, in comparison to the giant size and the latest technology adopted for LHC. However, an attempt has been made to share some experience discussing the technology involved earlier in the selection of power supply requirement for high energy particle accelerators. This review may help to understand the advancement of technology over last 60 years which is necessary at this stage for two reasons. Firstly, Saha Institute of Nuclear Physics, a pioneer research institute in India, has taken up an ambitious project for the installation of third generation synchrotron in India and secondly, this paper will open up research and developmental possibilities in the field of applications of the pulsed power technology in the areas of:

1. Industrial applications,

2. Scientific applications,

3. Open up new technology for high power switching,

4. Open up new concept in the design of $\mathrm{Rf}$ power supplies,

5. Develop new field for sophisticated detectors

6. Develop new methods for modeling and computing techniques.

Experiments with particle physics require high kinetic energy to be imparted to the charged particles. The development of very high kinetic energy of the particle is required for the bombardment at the target and thereafter record, and evaluate the result of the disintegrated particles from the collision to attain at some conclusion. How 
charged particles are to be generated, how such high kinetic energy can be imparted to the charged particles, how the result of the disintegrated particles after the bombardment can be detected, recorded, evaluated, interpreted and how all such requirements can be practically implemented is a total team work.

\section{Basic Physics and Process Flow Sheet of Protons}

One of the most powerful accelerators as regards the energy of the particle is the proton synchrotron. As mentioned earlier, imparting highest kinetic energy to the charged particle is important and this is being achieved through several stages as mentioned below;

1. First stage is the production of charged particles through Cockcroft Walton Generator.

2. Second stage is the initial acceleration through linear accelerators.

3. Third stage is the injector part, where the accelerated charged particles are injected into the desired position of the Proton synchrotron.

4. Fourth stage is the circular accelerator or the synchrotron, where the centrifugal force of the particle must be equated to the Lorentz force to maintain the circular path of radius $\mathrm{R}$ unchanged. Briefly the equations given below are important to understand the operation of the Synchrotron accelerator and for further reading on this subject text books may be referred.

$$
\begin{aligned}
& \mathrm{mv}^{2} / \mathrm{R}=\mathrm{evB} \\
& \mathrm{R}=\mathrm{mv} / \mathrm{eB} \\
& \mathrm{f}=\mathrm{v} / 2 \pi \mathrm{R}=\mathrm{eB} / 2 \pi \mathrm{m}
\end{aligned}
$$

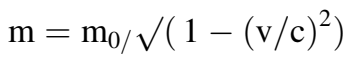

where, $\mathrm{R}$ is the radius of the circular path; $\mathrm{B}$, the magnetic flux density; $f$, the frequency of accelerating voltage; $\mathrm{C}$, the velocity of light $\mathrm{e}=1.6 \times 10^{-19} \mathrm{As}$; and $\mathrm{m}_{0}=1.67 \times 10^{24} \mathrm{~g}$;

5. Fifth stage is the extraction part of the beam to be bombarded at the target.

6. Preparation of the target area; to detect the disintegration after collision, record, evaluate the data and interpret the result of the experiment.

In proton linear accelerator, the particles develop their initial kinetic energy as per the design of the PLA and afterwards it is injected into the torus of the proton synchrotron, where the particles are accelerated further during each revolution. At the time of acceleration in synchrotron, some important operational conditions are followed;
1. Particles are not allowed to attain their full acceleration immediately after injection and therefore, the voltage necessary to raise the acceleration to maximum has to be imparted slowly in the form of voltage 'kicks' during each revolution, in other way, the particles should not pass through the accelerating voltage at once.

2. Particles are allowed to make rotation several times in succession within a very short period to develop the energy from the potential field.

At Rutherford High Energy Laboratory (RHEL) the first Linear Accelerator (Linac) was installed in 1957. The Proton Linear Accelerator (PLA), a $50.0 \mathrm{MeV}$ machine was developed initially in stages as a three tank unit at RHEL and the stages were as follows;

$$
\begin{aligned}
& \text { First tank-0.5-10 MeV } \\
& \text { Second tank-10.0-30.0 MeV } \\
& \text { Third tank-30.0-50.0 MeV }
\end{aligned}
$$

In RHEL, the PLA produced the first beam in July 1959. Later on it was again upgraded with the addition of the fourth tank to $70 \mathrm{MeV}$ to raise the injected energy level higher for further research work.

\section{Engineering Design Considerations}

\section{Civil Engineering}

Civil engineering, along with other engineering services during project handling played a significant part in the civil design and foundation of Nimrod along with associated equipments. The primary constraint of the civil design was the safety of personnel and equipments. When the synchrotron would be operational, the protection from intense radiation produced would be of serious concern and therefore, the civil design was to ensure that the injector and magnet rooms were adequately shielded to provide necessary safety in all respects. The effect on the temperature of the surroundings due to heat generated from various machine components in an enclosed area and adequate shielding for the protection of instruments from background radiation was given due importance in the design. In view of that, semi-ground construction was adopted for the main building and both concrete and earth were employed for shielding.

\section{Mechanical Engineering}

Design and manufacturing of magnet sector plates was a highly skilled job and without going into too much of mechanical detail it can be mentioned that the manufacturing of electromagnet for $7 \mathrm{GeV}$ proton Synchrotron was 
another engineering marvel. The ring shaped $7000 \mathrm{t}$ electromagnet had $48.768 \mathrm{~m}$ diameter and consisted of equal core shaped sectors and disposed in a circle comprising eight octants. The magnet sectors were fabricated at the Wolverthon works of M/s Sankay of England and for ease of transportation from the manufacturer's works site to the project site of RHEL, at Chilton, Berkshire, it was developed in pieces of 336 sectors. Each magnet sector was built of forty-five silicon steel plates, $3.17 \mathrm{~m}$ high and $2.82 \mathrm{~m}$ wide. With regard to high standard of specification demanded, manufacturing of magnet plates and its alignment played a special roll. Sufficient precautions were taken during manufacturing process to meet the highest tolerance requirement specified for each activity with regard to annealing, shearing, drilling, and machining at the factory. A special procedure was evolved to ensure that the surface finishes of the plates were of highest standards. The proper installation and positioning of these magnet sectors at the project site and their alignments, apart from all other activities had to undergo stringent inspection and scrutiny to satisfy the challenging technical demand. The geometric alignment of the magnet of the proton synchrotron presented unusual problems at the time of installation and commissioning because of accuracy required to maintain the plane of rotation during commencement and completion of bursts of pulses otherwise, the accuracy would have been affected due to sinking or tilting of the magnet foundation.

\section{Electrical Engineering}

\section{Selection of Magnet Power Supply}

Nimrod produced a high speed beam of protons at an energy level of $7 \mathrm{GeV}$ which was then bombarded on targets arranged as per the requirement of the experiments. Resultant nuclear reactions were then studied to provide data on the behavior of elementary particles. In addition to Nimrod with its demand of currents up to $10,500 \mathrm{~A}$ at $15 \mathrm{kV}$, other equipments such as Proton Linear Accelerator and RF generator designed to give the required voltage kick to boost up the energy level during each rotation of the particles made a heavy demand on the power supply equipment. Energies of about $15 \mathrm{MeV}$ from the PLA completed with injector would be injected into a large evacuated torus of the synchrotron. These particles would be further accelerated by HF electrostatic impulses imparted at regular intervals around the torus. Nimrod, was designed to produce pulses of $7 \mathrm{GeV}$ protons at a rate of about 28 pulses/min with about $10^{12}$ protons in each pulse, this is equivalent to a mean proton current of about $0.1 \mu \mathrm{A}$ whereas, the $25 \mathrm{GeV}$ proton synchrotron of CERN was designed for maximum flux density of $1.2-1.4 \mathrm{wb} / \mathrm{m}^{2}$, acceleration cycle of $12-20 \mathrm{per} /$ min and anticipated protons about $5 \times 10^{10}$ in each pulse. Since Nimrod required pulses of $15 \mathrm{MeV}$ up to $0.002 \mathrm{~s}$ long duration at $2 \mathrm{~s}$ intervals, the energy from the injector had to be pulsed. The RF accelerating unit imparted 5-7 keV energy kicks to the protons on each revolution and after a period of $0.7 \mathrm{~s}$, by which time the protons would make almost 1 million revolutions to reach the desired $7 \mathrm{GeV}$ energy. Power consumption to the accelerator cavity was of the order of $1 \mathrm{MW}$. During each revolution when the energy of the protons were increasing, the magnetic field had to increase in proportion and in synchronism with the voltage kick to a peak value of $1.4 \mathrm{wb} / \mathrm{m}^{2}$ in order to confine the protons into their circular orbit of radius $\mathrm{R}$. When the protons reached the maximum energy, the beam was taken out with the help of extraction unit to strike in a target. After that, the magnetic field was gradually reduced to 'zero' and kept in readiness for injection of a further pulse. The whole process was to be repeated again. The nature of the demand of the magnetic field during each pulse from the starting of current rise to current zero is shown in Fig. 1. So far, it has been briefly explained how the protons were injected to the torus of the synchrotron magnet and the requirements of varying magnetic field with the application of proportional voltage kicks to maintain the proton in its orbit of radius R. But, the complexities involved in the design and development of electrical system along with associated electrical circuit to follow the desired characteristics posed a difficult hurdle to the electrical engineers and it needs to be highlighted to show a glimpse of the enormity of the power supply requirement for the magnet as well as for other critical equipments during that period. Present day engineers would not be able to appreciate this problem as most of the technologies adopted earlier to solve the hurdle have become obsolete, even the text books are not available for consultation and the courses are withdrawn from the syllabus long back. Although engineers engaged in similar jobs today, may not find it interesting to know the problems faced earlier and the discussion on outdated technology may sound futile but, they would definitely appreciate how the system approach being same the advanced technology can simplify the complex design. It is therefore, thought worth to record the history to understand the technical challenges of the earlier period. This paper will make an effort to highlight the developments in stages and the hurdles faced by the engineers to reach at the solution with the available technology of the time.

In this paper the emphasis on the discussion will be placed on the $7 \mathrm{GeV}$ proton synchrotron-Nimrod of RHEL but, only at times, a reference will be made to CERN's $25 \mathrm{GeV}$ Proton synchrotron, which was operational during the same period. Actually, there cannot be any comparison between the two units as the difference in the design features of the two synchrotrons was prominent 
Fig. 1 Magnetic field characteristics of Nimrod (with permission from Rutherford Appleton Laboratory, England United Kingdom)

\section{PULSE DIMENSIONS.}

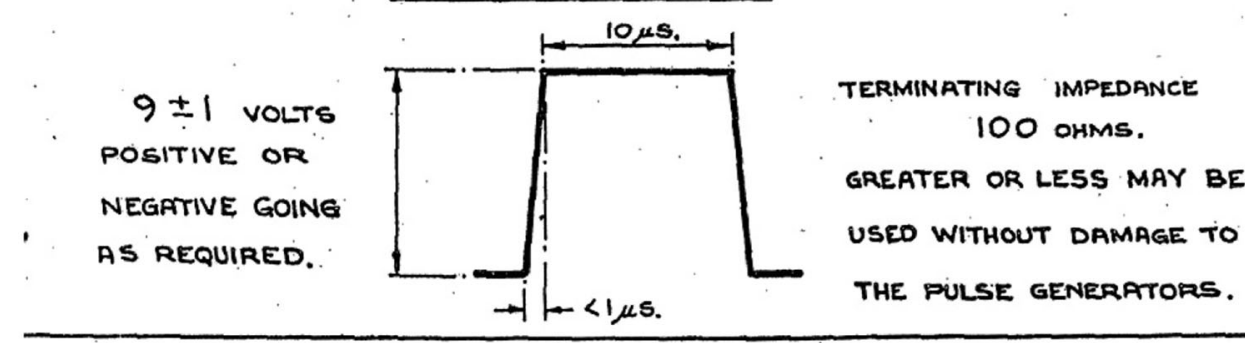

\section{SINGLE PULSES:}

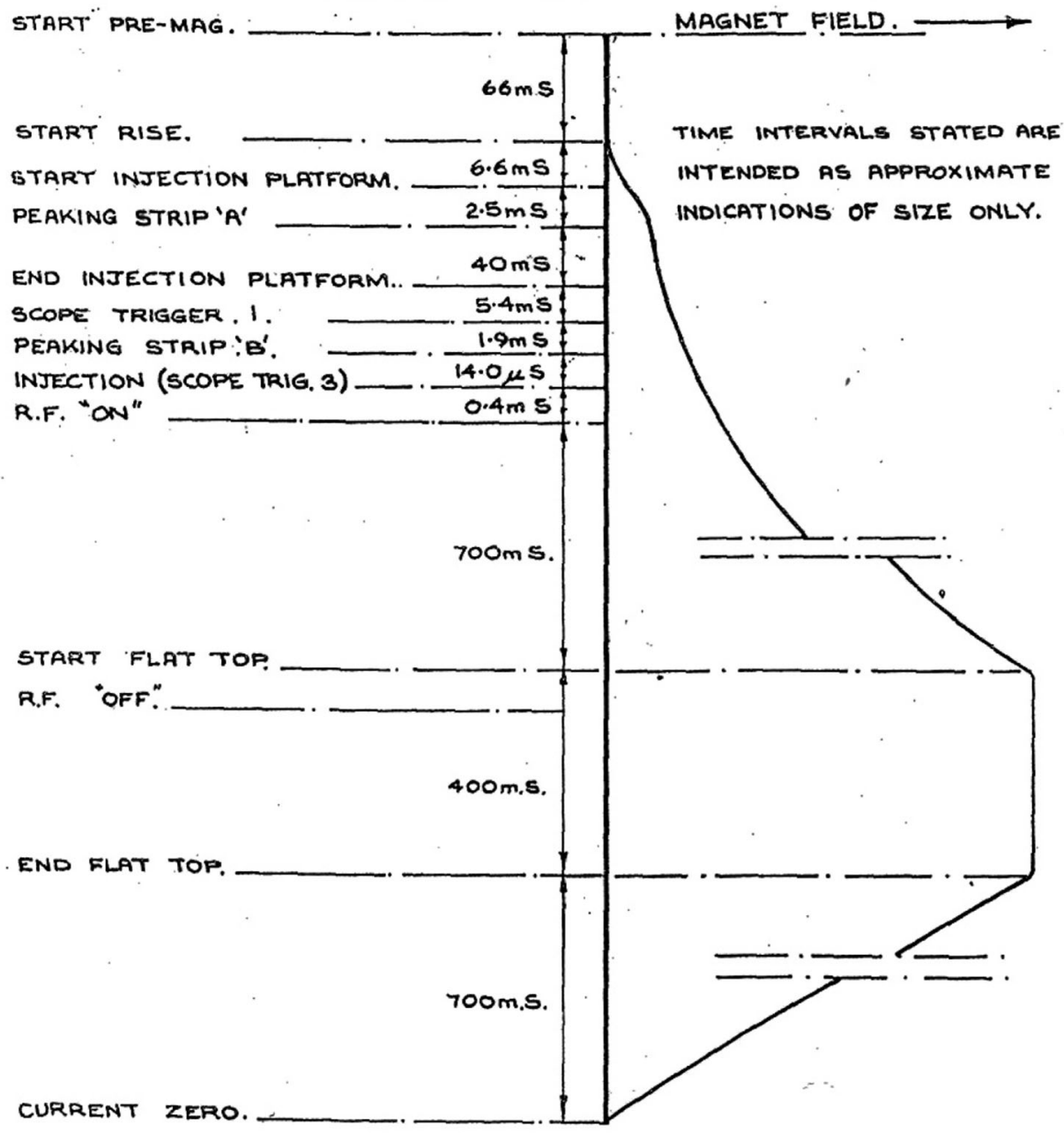

for various reasons. Firstly, the energy levels of the two units were different, secondly, the difference of energies of injected proton beam were also different, thirdly, the CERN's design was an earlier model than RHEL's Nimrod, fourthly, the difference in the selection of redundancy provision to maximize the operational efficiency of the synchrotron and thereby reduction in the loss of experimental time. Fifthly, separate converter configurations to contain the harmonics level and lastly, the most essential of all was the involvement of cost of installation during different period of times. It is only to be noted that, the problem in both cases was almost same. The very simple reason the project was challenging to the electrical engineers was that, the steep demand of current during energizing of the magnetic field within a very short period, and afterwards recovering of the energy stored in the magnetic coil for the utilization in the next. Table 1 presents characteristics of the proton synchrotron unit.

Figures 2 and 3 bear three elements characteristics. Figure 2 shows the characteristic curve for Nimrod and the Fig. 3 shows the characteristic curve for CERN synchrotron. 
Table 1 Characteristic detail of the two proton synchrotron units

\begin{tabular}{lllllllc}
\hline & $\mathrm{kV}$ & $\mathrm{kA}$ & Time $(\mathrm{s})$ & Time $(\mathrm{s})$ & Time $(\mathrm{s})$ & $\mathrm{c} / \mathrm{s}$ & $\mathrm{rpm}$ \\
\hline $7 \mathrm{GeV}$ Nimrod & 11 & 10.0 & 0.75 & 0.12 & 0.80 & 28 & $970-930$ \\
$25 \mathrm{GeV}$ CERN & 5.4 & 5.0 & 1.0 & Very low & 1.0 & $12-20$ & $3200-2800$ \\
\hline
\end{tabular}

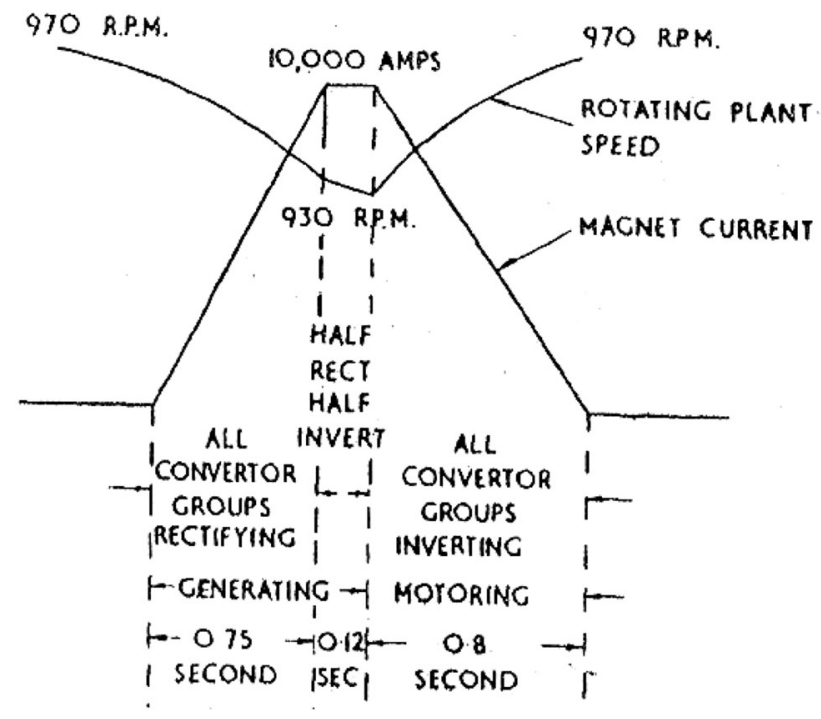

Fig. 2 Characteristic curve of nimrod power (with permission from Rutherford Appleton Laboratory, England United Kingdom)

Actually, the three elements characteristics as shown in the figures are very common in variable speed drive applications, such as, reversing rolling mills, mine winder drives and many other process applications but, the difference lies in the exacting demand of voltage and current during acceleration period and decaying period. The common features in both cases of characteristics are that, the first part is the accelerating part in case of variable speed drive, whereas, in case of synchrotron accelerator the magnetizing current is gradually rising. The actual time of current rise is depending on the value of magnetic field required. The second part is the flat top part, or the steady state part of the speed in case of variable speed drive, whereas, in case of synchrotron accelerator, it is the period during which time the magnet current is held constant. This condition is obtained by arranging only half of the converters to operate as rectifiers whilst the remainder functions as inverters. The third part of the characteristic in case of variable speed drive, is the decaying part and during this part, breaking is applied properly so that, the speed of the machine is gradually reduced to halt at the required position and ready for the next cycle to start without any difficulty. But, in case of synchrotron accelerator, it is the magnet de-energizing part. During this part the energy stored in the magnet coil is withdrawn and returned back to the supply. During this period, all the converters will be in

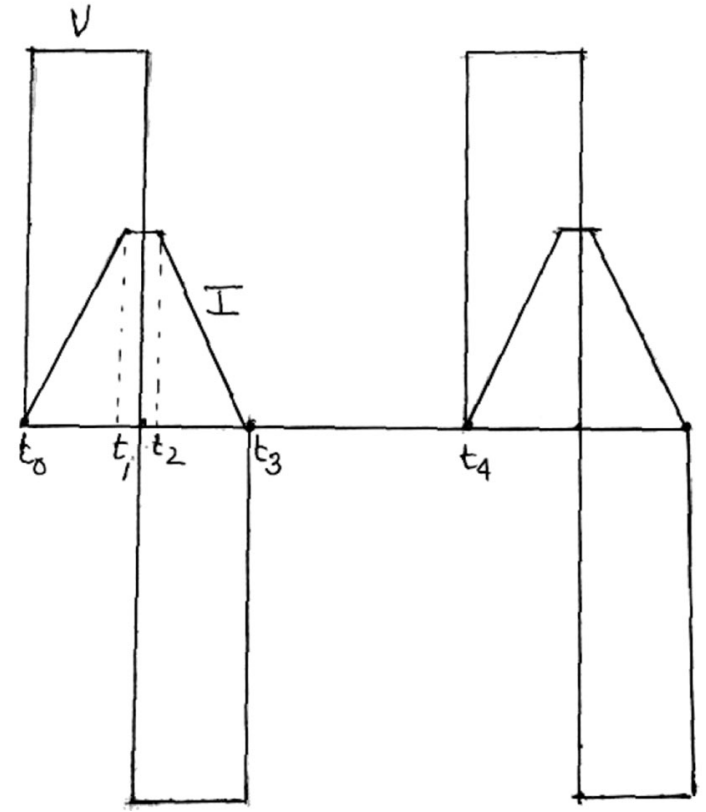

Fig. 3 Characteristic curve of CERN power (with permission from The Brown Boveri Review, Switzerland)

inversion mode. In case of variable speed drive, the factors mentioned above can be varied according to the demand of the process whereas, such variation is restricted in synchrotron application. The critical demand is that, the magnet must follow the set reproducible programme which exactly means that, after every pulse, the magnetic field has to come back to its initial state. The restoration of magnetic field to an initial definite state is essential after every pulse for synchrotron so as to perform a repetition rate of 28 cycles/s for Nimrod and 18 cycles/s for CERN's, synchrotron. Of course, the repetition rate for accelerators also can be changed with the change in the reproducible programme as per the requirement of experiment or the capability of the machine. The return to the initial state is governed by a factor known as remanence of the magnet material. A definite reproducible initial stage can be attained only when de-energisation of the magnet always follow the same curve. It is also important for the voltage at the magnet really to be zero during the pause period in order not to affect the initial state due to slight change in flux and therefore, subjected to greater tolerances. Otherwise, the protons would depart from their prescribed orbit soon after injection. In fact, the magnet voltage also must remain constant during energisation and maintain the 


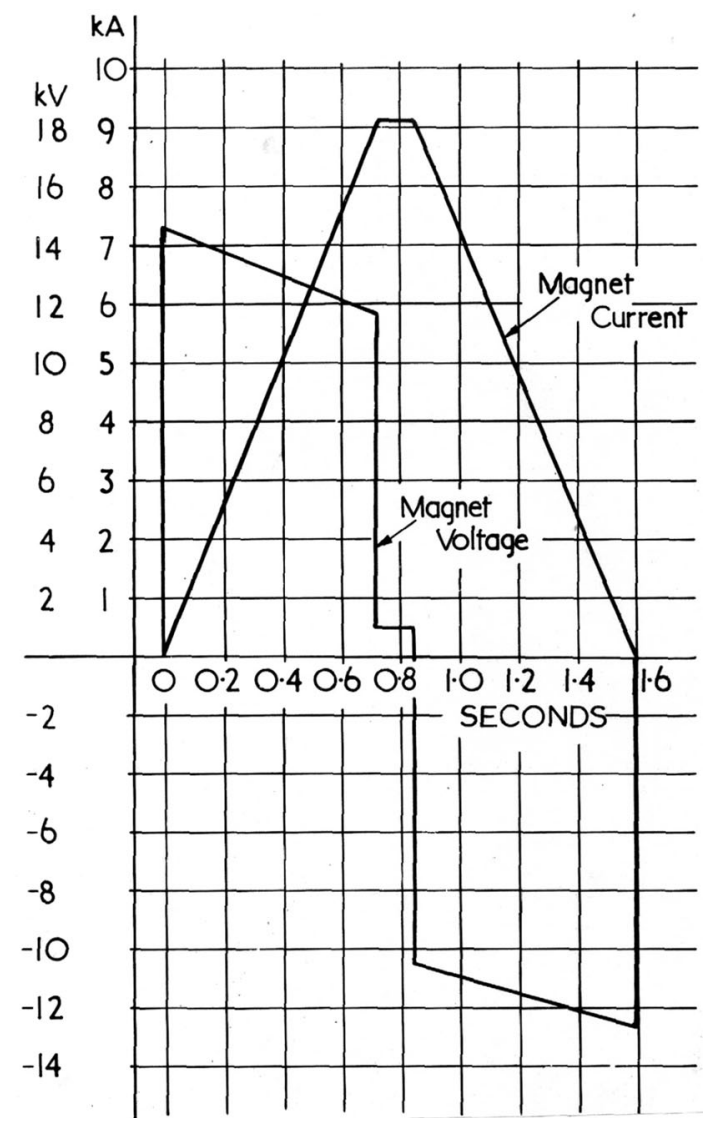

Fig. 4 Simplified pulse waveforms of magnet current and voltage Nimrod (with permission from Rutherford Appleton Laboratory, England United Kingdom)

reproducibility of the pulses within close limits. In Fig. 2, for Nimrod, the machines were acting as generators when the speed of the rotating plant falls from 970 to $930 \mathrm{rpm}$ and during this period the converter groups are acting as rectifiers. Obviously, the CERN's requirement showed the flat top portion to be nearly zero, and it was almost triangular in shape. Since the magnet from the electrical point of view behaves almost as a pure inductance, the stipulated current rise is obtained automatically if the applied voltage is kept constant. Simplified current and voltage pulse waveforms of Nimrod Magnet during operation is shown in Fig. 4. Also, a simplified characteristic of the alternator loading during operation is shown in Fig. 5. In case of Nimrod, the problem is to charge an induction of $1.06 \mathrm{H}$ with a maximum current of $10,000 \mathrm{~A}$ in a preset time of $0.75 \mathrm{~s}$.

After knowing the stringent demand of the magnetic field and the difficulties of pulsed power supply to the magnet, it may be assumed that, possible solutions to the problems might have been looked into from three available choices;
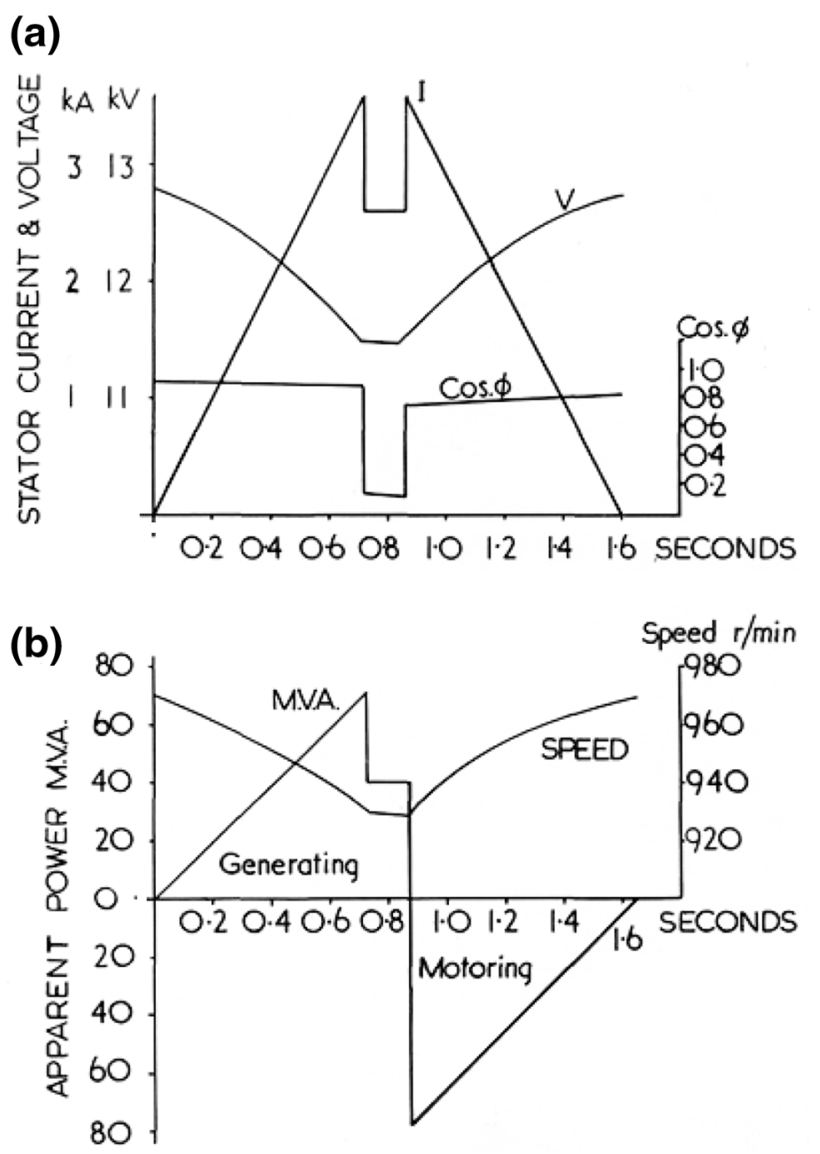

Fig. 5 Simplified characteristics showing alternator loading during a typical pulse (with permission from Rutherford Appleton Laboratory, England United Kingdom)

1. Supply the magnet power from a dc source.

2. Utilize ac to dc converter i.e. Supply ac power directly to the rectifier unit and feed the dc to the magnet.

3. Supply the ac power to a motor-alternator set and then convert to dc through rectifier before feeding to the magnet.

In case of 1 , in feeding the magnet from dc machines, the need for switching the magnet voltage on and off physically makes this difficult. Mechanical switching was ruled out by the frequent switching operations involved in pulse operation. It was only possible to connect or disconnect the dc voltage through the excitation of the machine but, the specified rise time of a few ms would have been difficult to attain by this method. Therefore, feeding the magnet direct from dc machine was ruled out.

The next possible method was to feed the magnet from converters directly connected to the supply network. In this case, the specified voltage rise would have been quite easily attained with grid controlled mercury-arc rectifier, but in that case, the supply network would have to possess a very large capacity to deliver the enormous power and 
Table 2 Specification of rotating equipments of power plants

\begin{tabular}{llll}
\hline & Alternator & Motor & Flywheel \\
\hline $7 \mathrm{GeV}$ Nimrod & $60 \mathrm{MVA}, 1000 \mathrm{rpm}, 3 \phi, 50 \mathrm{c} / \mathrm{s}, 11.5 \mathrm{kV}$ & $5000 \mathrm{hp}$ & $30 \mathrm{t}$ \\
$25 \mathrm{GeV}$ CERN & $46 \mathrm{MVA}, 3000 \mathrm{rpm}, 3 \phi, 50 \mathrm{c} / \mathrm{s}, 6.9 \mathrm{kV}$ & $2600 \mathrm{~kW}$ & $\mathrm{~N} / \mathrm{A}$ \\
\hline
\end{tabular}

withdrawal of stored energy from the coil during each pulse would create problem. It would also prove rather difficult to keep the specified constancy of the voltage and comply with the conditions imposed regarding allowable voltage fluctuation in the supply network. So this method was also ruled out.

There remains therefore, the third method where the magnet was to be fed from ac motor-generator set followed by rectifiers. This arrangement should be able to comply with the requirements on the power engineering side, only the contribution of the fluctuating load on the supply network needed to be seriously considered before the final design. Since the magnet windings form a highly inductive circuit, a large amount of energy would be stored in the magnet coil after withdrawal of the current. Hence, the withdrawal of energy from the coil and storing the energy in a technically feasible manner was important and accordingly, the application of flywheel in combination with the motor-generator was considered. The addition of flywheel would definitely control the fluctuating load to some extent in the input supply. In such cases, harmonic generation originated from the rectifier circuit would be made acceptable to the supply authority through proper configuration of the rectifier network to limit the harmonics to the specified limit.

Flywheel coupled to the motor generator set was common in industrial application as an energy storage device, even in some cases during emergency or interruption of power this stored energy is utilized as an uninterrupted source of supply for a very short duration. In synchrotron accelerator applications when peak and mean input power are compared, it will show how extraordinarily severe the conditions are for the power supply network to tolerate such fluctuating load. Such demand of fluctuating load can only be reduced with the help of energy storage units. The system must be such that, it not only be able to draw energy from the storage source during magnetizing part but be able to accept the return of energy when the magnets are deenergized. The storage unit must therefore be so designed that it supplies as well as accept the return of large amounts of energy from the magnet while, the input power from the supply network will be drawn only to meet the losses. Therefore, to meet the pulsating demand for the accelerator application, the combination of a flywheel coupled to motor and generator set in the same shaft was adopted.
Incorporation of flywheel set to absorb or deliver energy mostly depends on the total mass and the diameter of the flywheel. The violent cycling in speed and load amounts to a typical fluctuation in speed from 970 to $930 \mathrm{rpm}$ in case of Nimrod, whereas, the speed variation was restricted to $3200 \mathrm{rpm}$ and $2800 \mathrm{rpm}$ for the CERN's installation. Again, the speed of the synchronous machine could not be allowed to fluctuate unduly otherwise, it will raise complications in control. The energies which could be obtained from the rotating masses are proportional to $\mathrm{WD}^{2}$ where, the diameter is selected from the difference between the squares of the maximum and minimum speed variation. Therefore, larger the speed difference, the smaller the flywheel. In order that equipment is kept to a minimum and that the least demand is made on the incoming electricity supply a flywheel weighing $30 \mathrm{t}$ had been coupled on each motor-alternator rotor shaft for Nimrod, the amount of energy circulated in this way amounts to about 45 MJ. The detail ratings of the electrical equipments in each organization have been shown in Table 2. Each alternator is rated at $60 \mathrm{MVA}$ at $12.8 \mathrm{kV}$ and weighs a total of $400 \mathrm{t}$.

In variable speed drive for rolling mill, mine winders and in many other processes, any one of the three methods outlined above are selected according to the requirement of application. But the most interesting point to be noted here is that, earlier in variable speed drive application, WardLeonard drive was common and the mercury-arc rectifiers were avoided. In all such application rotating machine was the only choice. But in case of synchrotron, the magnitude of current and voltage to be handled required some serious analysis before the selection of a suitable design out of the three available methods. The actual constraint was that, any deviation in the supply characteristics will create a serious problem to hold the proton in its orbit and jeopardize the experiments. The consequences of such demand of pulsating load to the electricity supply network, in other words, restrictions imposed by the local supply authority on the fluctuating loads and the installed electrical equipments needed critical examination before the selection of the system.

A group consisting of 96 mercury-arc rectifiers for twin set of Nimrod and 24 mercury-arc rectifiers for CERN's with a huge rectifier plants with all its accessories, and control systems operating in open space made a special impact on excellent mechanism of technology. 


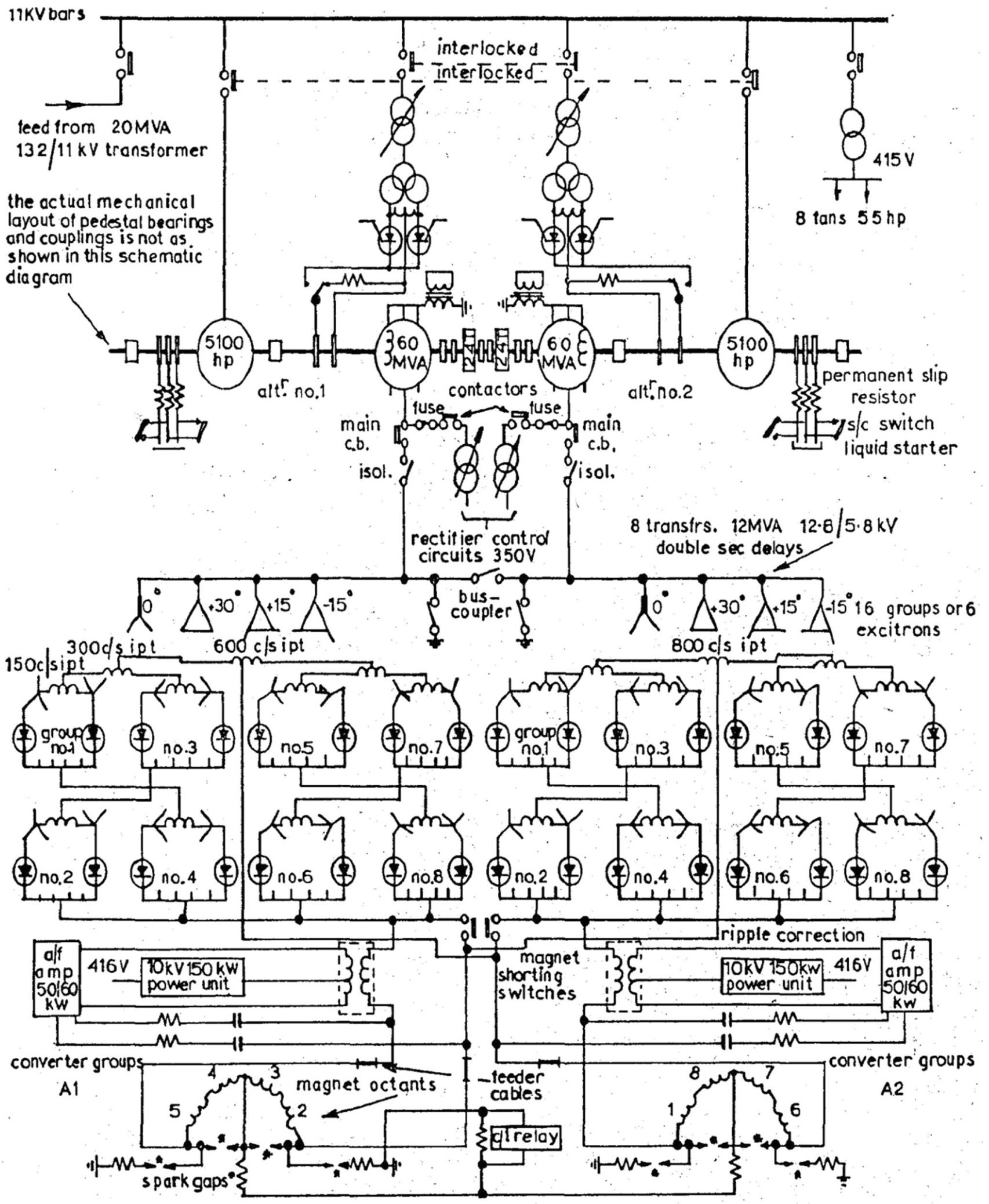

Fig. 6 RHEL power plant schematic diagram (with permission from Rutherford Appleton Laboratory, England United Kingdom) 


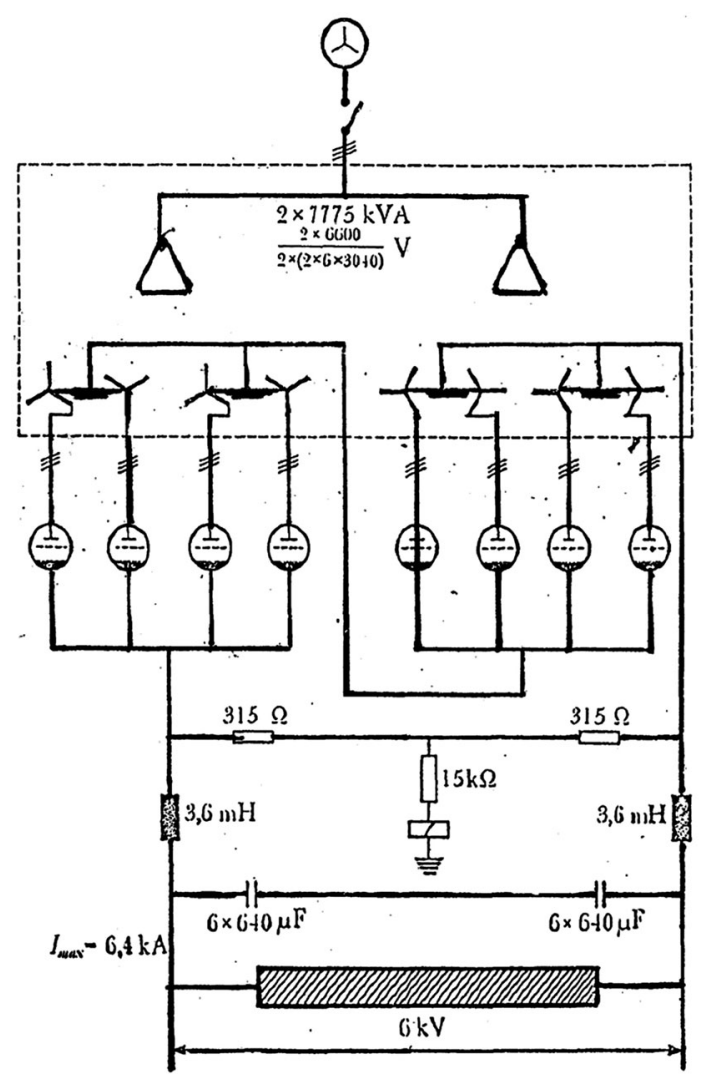

Fig. 7 CERN power plant schematic diagram (with permission from The Brown Boveri Review, Switzerland)

The layout of the complete installation and the basic features of the synchrotron power plants of the two units are shown in Figs. 6 and 7.

The system design was basically same for both the organization but, the difference was on the selection of equipments, choice of electrical systems, philosophy towards providing maintenance support, cost factor, factor of safety of equipments and some other factors. The two synchrotrons had their specification different including the injector energy input to the machine. Therefore, it was natural that the selection of equipment ratings, flywheel size, transformer connections and the rectifier configurations would be different. The most prominent part was the selection of speed of the set, more clearly, one unit selected two pole machine whereas, the other unit selected six pole machine. It may be seen that the diameters of the flywheels were also of different sizes. Since the diameter of the flywheel is dependent on the square of the difference of the speed variation during pulse period, the dominant factor is the tolerable variation in speed during operation. But, at the same time, during the selection, the importance had to be given to restrict wide variation of the speed; otherwise, it would have serious effect on the supply network. Nimrod was a very expensive machine and therefore, it was designed to operate at its maximum efficiency. From the design, it was clear that RHEL had given due importance on the redundancy of the power supply to the magnet. The power supply to the Nimrod proton synchrotron was effectively in two parts.

This twin arrangement of motor-generator-flywheel set was independent and provided some stand by protection against any serious breakdown on any one of the rotating equipment. In case of breakdown in any one unit, the operation on the other half could be continued to maintain power supply at reduced load so that, it could at least serve some experiments to continue. But, in case of CERN's power supply arrangement, redundancy factor was not taken into consideration, there could have been number of reasons for such decision but few possible reasons might be (a) the cost factor because, the cost of the dual set would have been exorbitant at that time or (b) it might be that the factor of safety chosen in the selection of equipments for the rotating machines were kept much higher to give adequate cushion for any breakdown or (c) it might be that, the experimental load was not expected to be high enough for which the breakdown time could not be spared.

With the massive weight of the rotating mass of motoralternator-flywheel set of about 400 tons and with the fluctuation of speed from 970 to $930 \mathrm{rpm}$ during each pulse, it was anticipated that, an alarming problem will be created with regard to vibration during operation of the Nimrod synchrotron. In order to restrict such tremendous vibration to a limited value adequate damping arrangements were made by mounting the total assembly on a reinforced and post stressed concrete foundation block weighing about $1200 \mathrm{t}$. Again, this foundation block was supported on 80 spring units and twelve viscous damper units. This foundation block ensured that virtually no vibration would transmit to nearby plant and buildings. Apart from the vibration damper arrangement mentioned, each set was provided with bearing vibration and shaft eccentricity measuring instruments. A view of the massive twin motor-alternator set combined with flywheels is shown in Fig. 8. At this point however, it may be appropriate to establish how true and effective the choice of dual set was in the electrical system design for Nimrod when the operational history of the Proton Synchrotron at RHEL is looked into. Actually, the first motor- alternator-flywheel set was installed and commissioned in conjunction with the converter plant during the early part of 1962 and the power supply was used for Nimrod magnet until August 1962. The second set was installed during the latter half of 1962 and commissioning was completed during February 1963. The synchrotron accelerator continued its operation until a major fault occurred on number 1 alternator in 1965. As per the site investigation report, the fault was due to failure of rotor pole end plates. Since the decision to adopt two 


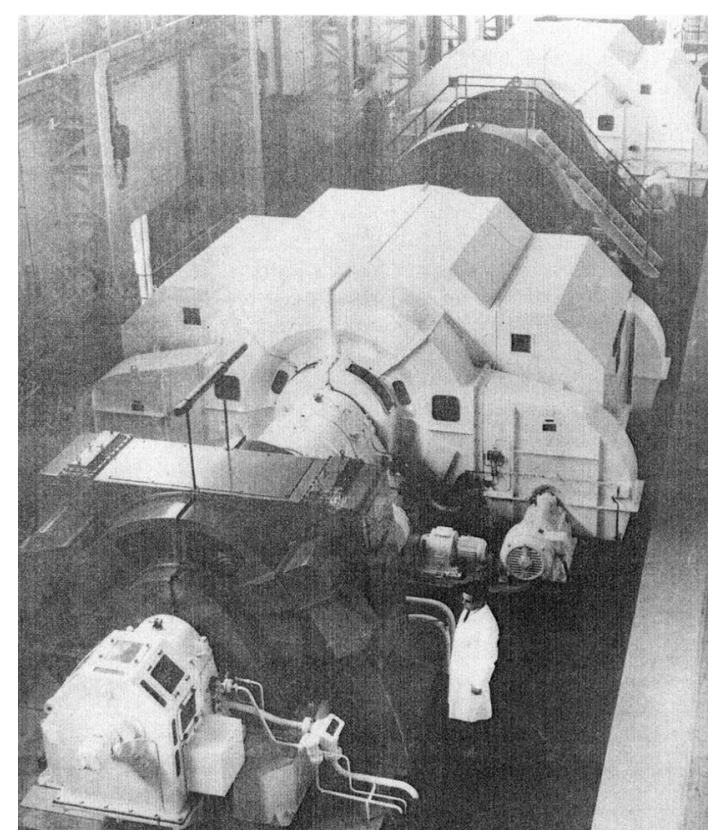

Fig. 8 Motor-alternator-flywheel sets (with permission from Rutherford Appleton Laboratory, England, United Kingdom)

sets was made and commissioned, the failure of one alternator could not totally disrupt the operation of the synchrotron. The synchrotron was kept operating even during this breakdown period of one set at lower capacity. The investigative work in connection with the cause of failure by the engineering team along with the manufacturer of alternator, took quite a long time. The rotating set was subsequently restored back to circuit for operation to continue after some modification in the design and strengthening of protection system. It could be noticed that the power requirement for the CERN's unit was much lower than the Nimrod's. This is evident from the choice of motors, alternators, the flywheel set, the choice of transformers connected to the rectifiers and the rectifier configurations [2].

\section{Transformer Connections and Selection of Rectifier Groups}

Before proceeding to the selection of transformer connections and rectifier configuration between the alternator supply and the connected load, the effect of harmonic distortion due to rectifier on a.c. supply was of greater interest and required serious consideration at the time of designing of such a system, as the rectifiers were among the largest harmonic producing devices connected to the supply. But, it was not possible to lay down a generally applicable definite limit of permissible harmonic voltage distortion on the point of common coupling, since the permissible value depended very much on the various items of plant and equipments connected to the supply system. However, the system design had to satisfy the strict stipulation regarding harmonics imposed by the supply authority. Apart from that, any load which varies rapidly in magnitude produces a corresponding variation in the supply voltage and because of this, the electricity boards had to introduce limits of voltage variations which they will accept at the point of common coupling for fluctuating load. A complete power plant schematic with the transformer and rectifier configuration adopted for Nimrod is shown in Fig. 6 and the same for CERN synchrotron is shown in Fig. 7.

As can be seen in the figure, each alternator feeds four rectifier transformers, each having a primary rating of 12 MVA. The primary windings of the four transformers are connected in star, delta, extended delta $\pm<15^{\circ}$.

Each transformer had two secondary windings connected double star with inter-phase transformer arranged as a twenty-four phase system. Each transformer secondary fed six single anode steel tank Mercury Arc converters of the water cooled continuously evacuated excitron type. As mentioned earlier and it can be seen from the Fig. 6 how rectifier groups are arranged. Eight groups with six converters in each group feeding to the four octants that is 48 rectifier load was connected to each motor-alternator set. The other motor alternator set supplied power to remaining four octants $(8 \times 6 \times 2=96$ converters). The reason for such dual connection was that, if any serious defect developed on one half of the power supply, the other half could still be used to pulse the magnet.

In case of CERN, two transformers were used in the primary side and the primary windings of the two transformers were connected in polygon, the secondaries being mutually displaced by 30 electrical degrees, so that output voltage of the rectifiers had a twelve phase ripple. Each transformer had two secondary windings in a double three phase connection with an inter-phase transformer for a dc voltage of $3000 \mathrm{~V}$ and a peak current of $6400 \mathrm{~A}$. In this case two groups having four sets in each group were arranged with three rectifiers in each set $(3 \times 4 \times 2=24)$. The outputs of the two units were connected in series as shown in the Fig. 7 to obtain the required voltage of $6.0 \mathrm{kV}$. The required dc voltage was produced by two series groups of converter units, comprised of 24 single anode steel tank mercury-arc rectifier. The converters were arranged in this way to reduce the harmonics in the $\mathrm{dc}$ voltage to minimum. In this case also single anode mercury-arc rectifier tanks were used with water cooled to obtain a temperature distribution as nearly uniform as possible. Six tanks formed a set and each set being mounted on its own framework insulated from earth with the necessary accessories for firing and excitation.

With regard to the choice of rectifier units, both the units had no other alternative but to choose single anode grid 
controlled mercury-arc rectifier as because Semiconductor rectifiers came little later. With the advent of semiconductor diodes, and controlled rectifiers (thyristors) from late sixties and with its various advanced forms, the design philosophy did changed. At the earlier stages of introduction of controlled rectifiers, some drawbacks were there in their application due to the limitation of power ratings, voltage ratings, current ratings, duration of turn-on, turnoff times, etc. Wider application of semiconductor devices was restricted but, from early seventies with the up gradation of power handling capabilities, improvements in dv/ $\mathrm{dt}$, di/dt characteristics, introduction of $\mathrm{I}^{2} \mathrm{t}$ fast acting fuses for protection, different combinations of circuit configuration with thyristor and diodes, the application of semiconductor devices for power control was universally accepted as best. Later on, the semiconductor diodes and controlled rectifiers with its various forms started dominating totally the application area. The converter and inverter grade thyristors or much better and improved version of semiconductor devices is now readily available for high power applications. Only interesting feature that had to be noticed in the design was the selection of the phase displacement of the transformers and their numbers as per the number of phase grouping of mercury-arc rectifiers. Actually, present day engineers would not be able to appreciate this problem as the technology used earlier became obsolete from early seventies. The practicing engineers may now find the solution to be simpler because of the easy availability of the power handling devices, the acceptable technology for reducing harmonics, simpler method of device cooling, suitable electronic control system for monitoring of various parameters.

\section{Electrical Supply to Support Equipments}

Earlier, it has been mentioned that this paper will briefly touch upon the power supplies to the critical equipments associated in the operation of Nimrod. Those were RF power supply, beam line magnets and bubble chamber supply.

\section{RF Power}

The protons were injected in the torus of Nimrod at an energy level of $15 \mathrm{MeV}$ with an orbital frequency of $355 \mathrm{k}$ $\mathrm{c} / \mathrm{s}$ and as the protons were accelerated to $7 \mathrm{GeV}$ energy this frequency was raised on a relativistic curve to $2.2 \mathrm{~m}$ $\mathrm{c} / \mathrm{s}$, corresponding to a velocity very nearly equal to that of light. The RF field was in synchronism with this. Without going into detail complexities involved in the design of R.F power it can be mentioned that it had three main sections;-
1. The high power RF equipment providing the accelerating voltage.

2. The low power RF equipment or primary frequency generator (PFG), providing an accurate and controllable frequency source for the high power RF system.

3. The beam control system, which provides a means of comparing the radial position and phase of the proton bunches with suitable reference value and of correcting any deviation through the PFG.

\section{Beam Line Magnets}

High energy beam from Nimrod are guided to various experimental areas by beam lines using quadruple and bending magnets. The power required for the beam line magnet can be said to be cost of transportation of the beam to the target. It will be surprising to know that the total power required to steer the beam to the experimental area was very high and almost comparable to the enormous power drawn for the production of the proton beam. A stabilized current supply to these beam line magnets was essential because any variation beyond the limit of $0.1 \%$ would result in improper steering and loss of particles. The total power requirement was of the order of $10 \mathrm{MW}$.

\section{Bubble Chamber Supplies}

In the collision of the extracted proton beam from Nimrod and the target, nuclei secondary particles were produced. These secondary particles were steered by the beam line magnets to the electrostatic separators where the so called 'bullet' was selected and separated. The bubble chamber recorded an 'event' after an interaction between the bullet selected by the separator and the liquids in the chamber. The supply to the bubble chamber magnets are from $4 \times 1 \mathrm{MW}$ dc sets.

\section{Power Distribution System}

Bulk supply was obtained from the Central Electricity Generating Board (CEGB) by two independent $132 \mathrm{kV}$ grid lines to the Atomic Energy Research Establishment, almost adjoining the Rutherford High Energy Laboratory. Supply was drawn to the RHEL from the common load point at the CEGB's grid compound. Actually, in addition to the magnet power, electrical power supplies were provided for the functioning of the inflector system for the beam entry to the magnet beam lines, plunging mechanisms, computer section, air conditioning, cooling and 
vacuum system for the synchrotron. In the proton synchrotron, a vacuum of $0.000133 \mathrm{~Pa}$ was usually maintained and such a high vacuum was essential to keep the loss of particles due to scattering by gas molecules down to an accepted level. Under operational condition the heat generated by various machine components in an enclosed area would raise the temperature of the surroundings. A nonuniform structural deformation of the magnet hall due to temperature rise of the surroundings might affect the alignments of the magnet sectors and consequently disturb the proton beam. In order to prevent structural instability, the magnet hall was air conditioned.

\section{Present Status of Synchrotron Accelerator- 'Rutherford-Appleton Laboratory'}

\section{ISIS Facility at Rutherford Appleton Laboratory}

The huge cost of such experimental set up for Nimrod as discussed above had to come to an end at some point of time, when the project Nimrod was terminated. It is quite natural that during the course of time the experimental need may change, and the future projects are required to be evaluated bearing in mind the cost involvement and the greater benefit to be gained from such experiments. In order to encourage and fulfill the aspirations and demand of the investigators, new frontiers of knowledge and research work were required to be opened up. After the official declaration of closure of the project Nimrod in 1977, the British researchers decided to shift the focus of the experimentation towards Spalled Neutron Source subsequently renamed as ISIS facility, which was set up at the Rutherford Appleton Laboratory (Rutherford High Energy Laboratory renamed as RAL) in the dismantled site of Nimrod to install the new accelerator necessary for such work. Proposal to set up spalled Neutron Source at the ISIS facility was taken up with a view to focus on the application potential of Neutron scattering and Muon spectroscopy in condensed matter and nuclear science. Subsequently, it was developed as the centre for one of the world's brightest neutron source. So far, the successes of ISIS are a result of the intense pulse of neutrons that the facility provides for experimentation. After the decision was made, the preparatory project work started to renovate the Nimrod project site and maximize the utilization of supporting equipments of Nimrod to reduce the project cost for the new synchrotron. In this context, it will be relevant to point out that before taking any decision on any new accelerator project, a serious consideration should be given on selecting suitable equipments, as the equipments in any large accelerator facility gradually becomes obsolete and the availability of spares becomes very difficult with the advancement of time because of the fast changing technology.

The life cost of such projects are to be evaluated critically with a fixed project time span and the future plans are to be reviewed time to time so that the course of research can be changed in a direction well in advance to get the maximum benefit from the research. Such decisions are very vital for the Indian scenario because here, with the delay in the project time not only the cost will accelerate but also the tuning of research topic will be shifted by the time it comes into operation. For instance, when ISIS facility was developed at the old site of Nimrod, some of the equipments were salvaged from Nimrod, even the magnet coil used in NINA synchrotron of Daresbury Nuclear Physics Laboratory, another unit of National Institute of Research in Nuclear Science (NIRNS) had been shifted to the RAL site to serve as the coil for the synchrotron under renovation and after that, the coil was referred as' Nina Choke'.

Actually, when a heavy metal target is bombarded by firing short bursts of accelerated protons of high energy, intense pulses of neutrons are produced. The process flow of proton beam is exactly same that had earlier been mentioned. Only the method by which such high energy is imparted to the protons by synchrotron accelerator is different. In this case also, the proton beams are injected into the synchrotron and then rotated around the ring and accelerated by RF cavities, focused and guided by a series of magnets. The field frequency is precisely double that at which the protons rotate around the ring so that they get an accelerating kick every time they go past a cavity. It also increases throughout the acceleration cycle to match the changing energies and rotational speeds of the protons. At the same time the protons are also squeezed into two bunches before being extracted and sent to the target station. In this case, the level of injected energy is $70 \mathrm{MeV}$ and the energy level of the ISIS synchrotron accelerator is of $800 \mathrm{MeV}$. At the heart of the ISIS accelerator is a proton synchrotron which is a large storage choke, 'Nina choke' as mentioned earlier, in the form of ring magnets with ten main windings connected in series with capacitor banks so that, they form a resonant circuit with fundamental frequency of $50 \mathrm{~Hz}$. Earlier, the method of direct excitation of the magnet was discussed but, during the course of time and in future projects the decision to switch over to resonant excitation of the magnet was an important deviation. The method of resonant excitation developed by White $[3,4]$ and known as 'white circuit' is one solution to reduce the load on the power supply and get rid of the massive fly wheel set. The cumbersome arrangements with large rotating equipments along with the associated rectifier plant to supply power to the magnet coil had been abandoned with the implementation of resonant excitation method. 
Earlier design had two hurdles; firstly, restoring the stored energy from the magnet coils during the decaying part and secondly, implementations of a suitable system for reducing the fluctuating load on the supply network. But, with the deployment of' white circuit' configuration, the problems are resolved in much simpler way. The concept of 'white circuit' has eventually eliminated the flywheel set which was so far essential to meet the fluctuating demand. It is a new aspiration for researchers in the field of nuclear physics but it is essentially a technological challenge to the engineers for the installation, operation, and maintenance of the highly sophisticated equipments with the latest art in design. It is expected that a high degree of sophistication will be involved and to appreciate and understand the implication of the demand, qualified and best trained engineers are to be engaged for the job. Any lacunae in the formation of such team would be disastrous for the project. Actually, the building and commissioning a synchrotron accelerator is a very lengthy process and this time factor plays an important part in deciding the areas of research because, by the time the system goes into operation the focus on the dominant area for research work may be diverted to other advanced field. So perhaps, in order to reduce the time as well as the cost of revamping, whatever could be salvaged from the remains of $7 \mathrm{GeV}$ proton synchrotron was retained for proper utilization for the ISIS facility. Even, in many cases, equipments now being used are as old as 50 years. Mainly, the PLA which was operating at $15 \mathrm{MeV}$ and the rest of the acceleration tanks that were in the pipe line during Nimrod operation as mentioned but, so far not taken into circuit, later upgraded with the addition of fourth tank to provide an injected energy of $70 \mathrm{MeV}$ to increase the energy level at the entry. At the time when ISIS spallation neutron source produced its first beam on 16th December 1984, it used $665 \mathrm{kV}$ CockcroftWalton pre- injector, a $70 \mathrm{MeV}$ Linac and $800 \mathrm{MeV}$ proton synchrotron with a cycling rate of $50 \mathrm{~Hz}$. It was decided that the PLA, which was later upgraded with the addition of fourth tank to $70 \mathrm{MeV}$ at the end phase of Nimrod, to be utilized as an injector to the synchrotron. With such changes in decision, the electrical engineering configuration shifted drastically. Earlier model of electrical supply to the magnet was replaced with the design concept of so called 'white circuit'.

\section{Further Modification Plan}

A synchrotron accelerator with "white circuit" configuration is shown in Fig. 9. In this case, the circuit is designed to resonate at the operating frequency of $50 \mathrm{~Hz}$ in order to reduce the reactive load on the makeup supply. It also has the advantage of ensuring that the same current flows in all

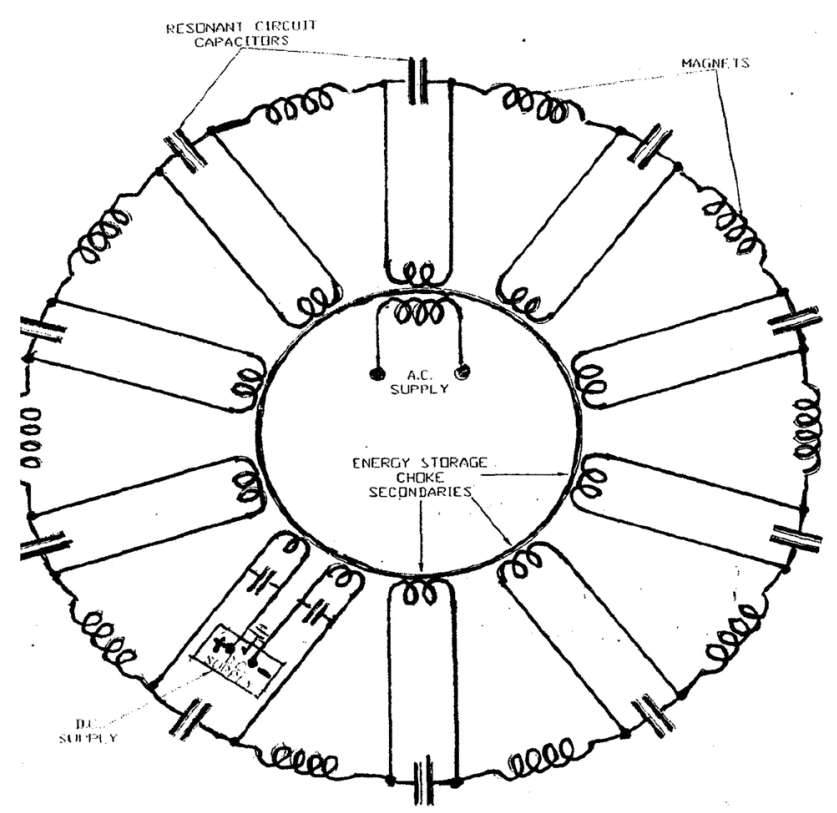

Fig. 9 ISIS Ring Magnets white circuit schematic (with permission from ISIS Facility, Rutherford Appleton Laboratory, England, United Kingdom)

magnets. Again, the way a dc supply is used to bias the sinusoidal magnet waveform can be seen. The dc bias is connected by splitting and centre grounding one element of the white circuit where as the ac make up power is supplied to the primary winding of the white circuit. The advantage of introducing a dc bias is to increase the efficiency of the white circuit by reducing the total power requirement for the same change in magnetic field from injection to peak energy. A very important consideration in the design of 'white circuit' is to maintain the resonant frequency. At the time of design it was expected that the value of the capacitor may fluctuate due to change in temperature and accordingly in order to check the fluctuation of capacitor value, half of the capacitor was chosen with negative temperature coefficient and the other half with positive temperature coefficient. But, during operation it was found that such gradation of temperature was not required as the ambient temperature due to heat generated could take care of the temperature equilibrium. Figure 10 shows how the ac is fed to the coil. In this case a motor-alternator set is used to supply the ac power but, the interesting part in the design is the conversion of $3 \phi$ alternator to single phase ac supply before feeding to the coil [5]. Actually, the windings of the Nina choke are built into a single frame and therefore closely coupled by the magnetic circuit but, the drawback with such arrangement is that, in the event of a failure of any winding the choke would have to be dismantled to replace the damaged parts. Therefore, the failure of any part of the coil could result in the shutdown for more than a year for rectification or replacement. The 
Fig. 10 The ISIS main magnet ac power supply (with permission from ISIS Facility, Rutherford Appleton Laboratory, England, United Kingdom)

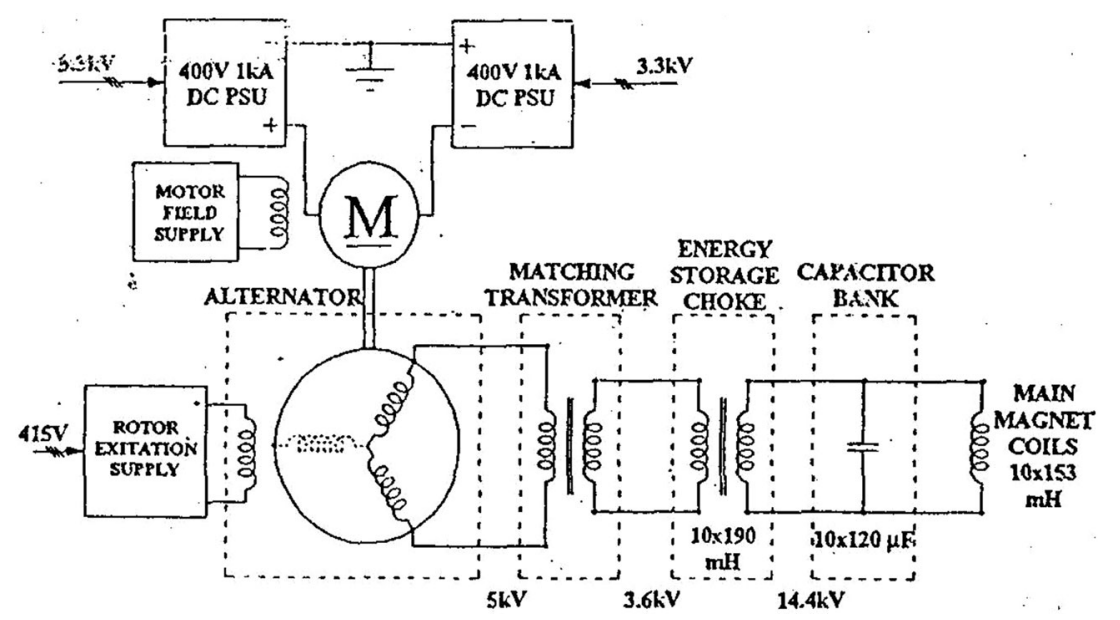

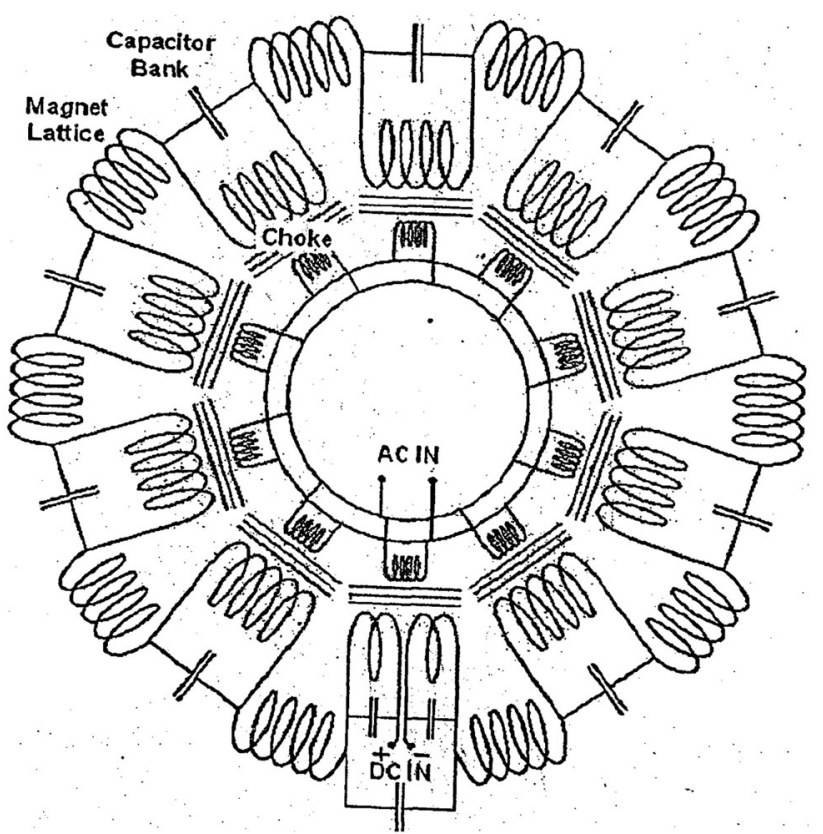

Fig. 11 Modified white circuit schematic (with permission from ISIS Facility, Rutherford Appleton Laboratory, England, United Kingdom)

problem at present in the operation of synchrotron is to find out a solution so that the long downtime due to breakdown in any part of the coil could be minimized before the replacement. Since the spares are becoming scarce a new approach to replace the coil within a reasonable time are getting priority in the design. In the replacement model the magnetic circuit is split into ten smaller chokes with main and auxiliary windings [6]. The design of 'white circuit' with modified choke is shown in Fig. 11.

Also, the processes of replacing the motor-alternator set with solid state uninterrupted power supply sources are being considered and the alternative arrangements are being tested. Three numbers of uninterrupted sources of supply are organized to supply the required power to the circuit. The upgrading of the ac supply to the main magnet is necessary to improve the system reliability, improving magnet current stability and reducing the risk of mains failure. The project site of RAL and the arrangements for new set up under test for up-gradation was shown and the critical problems arising out of the electrical design was discussed with the Chief Engineer when their plan to handle the technical difficulties was opened up.

\section{Conclusion}

The power system [7] plays an important part in the operation of high energy particle accelerators, the system design as well as the maintenance standards are kept high and strictly followed. Routine checking of technical parameters of all critical equipments laid down by the manufacturers and the technical cell reduced the breakdown to minimum. In order to give the maximum machine availability to the researchers the team work and the coordination was of the highest order. The experience from the past has been shared with a hope that, the future course of action for the synchrotron programme may be steered in a direction which will give positive results. The huge cost of experimental set up for scientific experiments to find out the ultimate particle in nature, must also be directed towards some result oriented experiments which will find a larger application area as mentioned earlier. Somehow, the cost-benefit has to be justified. Documentation and preparatory work for the installation and commissioning of a third generation state of the art high energy synchrotron facility in India has been initiated by Saha Institute of Nuclear Physics, Kolkata with the objective of providing research investigation on the material science which will help in medical applications to nano-technology in future. In such projects, time is an essence and since it has a high application potential, no bottleneck should come on the 
way to development otherwise, advanced research programme will always trail behind others. Also, here lies greater scope for research and development in the super conducting magnet systems which will open up a vast application field. The high magnetic field is required for accelerator operation which demands high currents to be supplied to the magnet. This process will allow a reduction in the circumference of the magnet ring which in turn will drastically reduce the cost of magnet. In order to achieve this goal superconducting materials capable of sustaining high current densities in magnetic fields are considered for potential application for high energy physics experiments. Now it has been possible to achieve high current densities in super conducting magnet systems which has been utilized in LHC to attain such incredible energy.

Acknowledgments The author would like to express his sincere gratitude to Rutherford Appleton Laboratory, Science and Technology Facilities Council, England, United Kingdom and The Brown Boveri Review, Switzerland, for giving permission to use from their sources some figures, diagrams and tables in the preparation of the technical paper without the support of such information the paper would never be completed.

\section{References}

1. P. Bowels, M.J. Marchbanks, Magnet power supply for the $7 \mathrm{GeV}$ protron synchrotron nimrod. Proc. IEE 110, 561-572 (1963)

2. R. Ludwig, Supply system for the magnet of the protron synchrotron of CERN. Brown Boveri Rev. 46(6), 327-350 (1959)

3. M.G.White et al., A 3 bev high intensity proton synchrotron the princeton-pennsylvania accelerator, CERN Symposium, 525-529 (1956)

4. M. Glover et al., Digital control system for the ISIS synchrotron main magnet power supply. Fourth European Particle Accelerator Conference, London, UK, 1830-1832, June 27-July 1 (1994)

5. J.W. Gray et al., Upgrade of the ISIS main magnet power supply. The European Particle Accelerator Conference, Vienna, 2202-2204 (2000)

6. M. Glover et al., Replacement of the ISIS white circuit choke. Proceedings of European Particle Accelerator Conference, Genoa, Italy, 3679-3681 (2008)

7. P.K. Dey, Distribution and utilization of electrical power at Rutherford laboratory. IEE S.Q.J 1-7 (1969) 